\title{
Magnetotransport properties of doped $\mathrm{RuSr}_{2} \mathrm{GdCu}_{2} \mathrm{O}_{8}$
}

\author{
J. E. McCrone, ${ }^{1}$ J. L. Tallon, ${ }^{2}$ J. R. Cooper, ${ }^{1}$ A. C. MacLaughlin, ${ }^{3}$ J. P. Attfield,${ }^{3}$ and C. Bernhard ${ }^{4}$ \\ ${ }^{1}$ IRC in Superconductivity, Cambridge University, Cambridge CB3 OHE, United Kingdom \\ ${ }^{2}$ Industrial Research Ltd., P.O. Box 31310, Lower Hutt, New Zealand \\ ${ }^{3} I R C$ in Superconductivity and Department of Chemistry, Cambridge University, Cambridge CB2 IEW, United Kingdom \\ ${ }^{4}$ Max-Planck-Institut für Festkörperforschung, D-70569 Stuttgart, Germany
}

(Received 27 February 2003; published 26 August 2003)

\begin{abstract}
$\mathrm{RuSr}_{2} \mathrm{GdCu}_{2} \mathrm{O}_{8}$, in which magnetic order and superconductivity coexist with $T_{\text {mag }} \gg T_{c}$, is a complex material which poses new and important questions to our understanding of the interplay between magnetic and superconducting order. Resistivity, Hall-effect, and thermopower measurements on sintered ceramic $\mathrm{RuSr}_{2} \mathrm{GdCu}_{2} \mathrm{O}_{8}$ are presented, together with results on a broad range of substituted analogs. The Hall effect and thermopower both show anomalous decreases below $T_{m a g}$, which may be explained within a simple two-band model by a transition from localized to more itinerant behavior in the $\mathrm{RuO}_{2}$ layer at $T_{\text {mag }}$.

DOI: 10.1103/PhysRevB.68.064514

PACS number(s): 74.25.Fy, 74.25.Ha, 74.72.Jt
\end{abstract}

\section{INTRODUCTION}

Soon after the first successful synthesis ${ }^{1}$ of $\mathrm{RuSr}_{2} \mathrm{GdCu}_{2} \mathrm{O}_{8}$, the material was found to display not only superconductivity $\left(T_{c} \simeq 45 \mathrm{~K}\right)$ but coexisting magnetic order with $T_{m a g} \simeq 135 \mathrm{~K}^{2,3}$ Evidence accumulated from static magnetization, muon spin rotation, ${ }^{3}$ and from $^{4}$ Gd-electron-spin-resonance ${ }^{4}$ studies showing that the magnetism is a spatially uniform bulk property. Specific-heat measurements ${ }^{2}$ and the diamagnetic shielding fraction at low temperatures ${ }^{3,5,6}$ indicate that the superconductivity is also a bulk property, and that the two phases therefore coexist on a truly microscopic scale. An initial neutron diffraction study eliminated the possibility of ferromagnetic (FM) order with the $\mathrm{Ru}$ moments lying in the $\mathrm{RuO}_{2}$ plane, but did not rule out FM alignment with the moments parallel to the $c$ axis, canted ferromagnetism, or itinerant ferromagnetism. ${ }^{7}$ Subsequent polarized neutron diffraction data ${ }^{8}$ have thrown the debate on $\mathrm{RuSr}_{2} \mathrm{GdCu}_{2} \mathrm{O}_{8}$ wide open by appearing to show that the underlying ordering of the Ru moments below the magnetic transition is in fact $G$-type antiferromagnetic (antiparallel nearest-neighbor ordering in all three crystallographic directions). Finally, more recent neutron measurements on $\mathrm{RuSr}_{2} \mathrm{YCu}_{2} \mathrm{O}_{8}$ confirmed that there is indeed a FM component of about $0.28 \mu_{B}$ which is about (1/5)th of the antiferromagnetic (AFM) component of $1.2 \mu_{B} .{ }^{9}$ The magnetic order shows a rather strong and unusual response to an applied magnetic field, with the FM component growing rapidly in strength and dominating over the AFM already at $2 \mathrm{~T}$. Whatever the nature of its magnetism, the discovery of this material is an exciting development which poses new and important questions to our understanding of the interplay between magnetic and superconducting (SC) order.

Magnetoresistance (MR), Hall-effect, and thermopower (TEP) measurements on undoped sintered ceramic $\mathrm{RuSr}_{2} \mathrm{GdCu}_{2} \mathrm{O}_{8}$ were presented previously. ${ }^{10}$ Above $T_{\text {mag }}$ the MR is negative and proportional to the square of the $\mathrm{Ru}$ magnetization and was ascribed to spin scattering of the carriers. A model for dilute magnetic alloys was used to extract a value $(\geqslant 25 \mathrm{meV})$ for the exchange interaction between the Ru moments and the carriers. Below $T_{\text {mag }}$ the Hall effect and TEP both fall anomalously. It will be shown that these data may be explained within a simple two-band model by a transition from localized to more itinerant behavior in the $\mathrm{RuO}_{2}$ layer at $T_{m a g}$. Evidence for delocalized carriers within the RuO layers has also been obtained from other transport and microwave absorption studies ${ }^{11}$ as well as from $\mathrm{Ru}-$ NMR (nuclear-magnetic-resonance) measurements where clear anomalies in the Ru-NMR relaxation rate occur near $T_{c} .{ }^{12}$ This suggests that the Ru nuclear moments experience a sizable hyperfine coupling to the charge carriers that enter the SC state.

The magnetothermopower reveals an extremely unusual variation of $T_{c}$ with applied field: ${ }^{6} T_{c}$ actually increases by $\sim 4 \mathrm{~K}$ as the applied field is increased to $2 \mathrm{~T}$. The increase saturates along with the Ru magnetization, suggesting that the onset of $\mathrm{Ru}$ magnetic order reduces a magnetic pairbreaking effect in the $\mathrm{CuO}_{2}$ layer.

The carrier concentration in $\mathrm{RuSr}_{2} \mathrm{GdCu}_{2} \mathrm{O}_{8}$ and its magnetic and SC properties, structural deformations, and so forth may be altered by cation substitution. Examining the transport properties of such samples should lead to a better understanding of the parent material. In this paper we present magnetotransport measurements on substituted $\mathrm{RuSr}_{2} \mathrm{GdCu}_{2} \mathrm{O}_{8}$. It will be shown that the data strongly support a simple two-band model in which the Hall effect and TEP of each sample are determined by the properties of the $\mathrm{CuO}_{2}$ and $\mathrm{RuO}_{2}$ layers, weighted appropriately by their conductivities. The model indicates that the $\mathrm{RuO}_{2}$ layer in the undoped material is very poorly conducting at room temperature, with $\sigma_{\mathrm{Ru}} \sim 0.1 \sigma_{\mathrm{Cu}}$, increasing to $\sim 0.3 \sigma_{\mathrm{Cu}}$ or higher at low temperature. While in most of the samples studied the $\mathrm{CuO}_{2}$ layer remains the better conductor at all temperatures, we find that the $\mathrm{RuO}_{2}$ layer dominates the conductivity below $T_{m a g}$ in a sample with $10 \% \mathrm{Ce}^{4+}$ substituted for $\mathrm{Gd}^{3+}$.

\section{EXPERIMENTAL METHODS}

Phase-pure sintered pellets of $\mathrm{RuSr}_{2} \mathrm{GdCu}_{2} \mathrm{O}_{8}$ were synthesized as described previously via solid-state reaction of a stoichiometric mixture of high-purity metal oxides and 
TABLE I. Substituted variants of $\mathrm{RuSr}_{2} \mathrm{GdCu}_{2} \mathrm{O}_{8}$ studied in this work.

\begin{tabular}{lc}
\hline \hline Composition & Substituted site \\
\hline $\mathrm{Ru}_{0.6} \mathrm{Sn}_{0.4} \mathrm{Sr}_{2} \mathrm{GdCu}_{2} \mathrm{O}_{8}$ & $40 \% \mathrm{Sn}$ for $\mathrm{Ru}$ \\
$\mathrm{Ru}_{0.8} \mathrm{Sn}_{0.2} \mathrm{Sr}_{2} \mathrm{GdCu}_{2} \mathrm{O}_{8}$ & $20 \% \mathrm{Sn}$ for $\mathrm{Ru}$ \\
$\mathrm{Ru}_{0.925} \mathrm{Sn}_{0.075} \mathrm{Sr}_{2} \mathrm{GdCu}_{2} \mathrm{O}_{8}$ & $7.5 \% \mathrm{Sn}$ for $\mathrm{Ru}$ \\
$\mathrm{Ru}_{0.975} \mathrm{Sn}_{0.025} \mathrm{Sr}_{2} \mathrm{GdCu}_{2} \mathrm{O}_{8}$ & $2.5 \% \mathrm{Sn}$ for $\mathrm{Ru}$ \\
$\mathrm{Ru}_{0.8} \mathrm{Nb}_{0.2} \mathrm{Sr}_{2} \mathrm{GdCu}_{2} \mathrm{O}_{8}$ & $20 \% \mathrm{Nb}$ for $\mathrm{Ru}$ \\
$\mathrm{Ru}_{0.9} \mathrm{Nb}_{0.1} \mathrm{Sr}_{2} \mathrm{GdCu}_{2} \mathrm{O}_{8}$ & $10 \% \mathrm{Nb}$ for $\mathrm{Ru}$ \\
$\mathrm{RuSr}_{2} \mathrm{Gd}_{0.8} \mathrm{Ce}_{0.2} \mathrm{Cu}_{2} \mathrm{O}_{8}$ & $20 \% \mathrm{Ce}$ for $\mathrm{Gd}$ \\
$\mathrm{RuSr}_{2} \mathrm{Gd}_{0.9} \mathrm{Ce}_{0.1} \mathrm{Cu}_{2} \mathrm{O}_{8}$ & $10 \% \mathrm{Ce}$ for $\mathrm{Gd}$ \\
$\mathrm{RuSr}_{2} \mathrm{EuCu}_{2} \mathrm{O}_{8}$ & $100 \% \mathrm{Eu}$ for $\mathrm{Gd}$ \\
$\mathrm{RuSr}_{2} \mathrm{Gd}_{0.6} \mathrm{Dy}_{0.4} \mathrm{Cu}_{2} \mathrm{O}_{8}$ & $40 \% \mathrm{Dy}$ for $\mathrm{Gd}$ \\
$\mathrm{RuSr}_{2} \mathrm{Gd}_{0.9} \mathrm{Y}_{0.1} \mathrm{Cu}_{2} \mathrm{O}_{8}$ & $10 \% \mathrm{Y}$ for $\mathrm{Gd}$ \\
$\mathrm{RuSr}_{2} \mathrm{GdCu}_{1.9} \mathrm{Li}_{0.1} \mathrm{O}_{8}$ & $5 \% \mathrm{Li}$ for $\mathrm{Cu}$ \\
\hline \hline
\end{tabular}

$\mathrm{SrCO}_{3} \cdot{ }^{3,13}$ The doped samples, listed in Table I, were produced similarly; the compositions given are nominal. A final extended anneal at $1060{ }^{\circ} \mathrm{C}$ in flowing high-purity $\mathrm{O}_{2}$ produces a marked improvement in the crystallinity of the undoped material, resulting in a higher resistive $T_{c}$ [as defined by $\rho(T)=0$ ] but no significant change in the thermodynamic $T_{c}{ }^{2}$

Bars of approximate dimensions $4 \times 1 \times 0.7 \mathrm{~mm}^{3}$ were cut from the sintered pellets using a diamond wheel, then polished down to a thickness of $\sim 150 \mu \mathrm{m}$ in order to increase the measured Hall voltage. They were mounted on quartz substrates in a standard six-contact configuration allowing both resistance and Hall voltage to be measured simultaneously. The contacts were made using $25-\mu \mathrm{m}$ gold wire and Dupont 6838 conducting epoxy, cured in air at $450{ }^{\circ} \mathrm{C}$ for $6 \mathrm{~min}$, giving contact resistances $<1 \Omega$.

Resistivity and Hall-effect measurements were made using an ac current source, low-noise transformers, and lock-in amplifiers. A frequency of $\sim 77 \mathrm{~Hz}$ was used to avoid mains pickup, with current densities of around $0.25 \mathrm{~A} \mathrm{~cm}^{-2}$. The Hall coefficient $R_{H}$ was usually measured by stabilizing the temperature and field (10 $\mathrm{T}$ unless stated otherwise), then measuring the Hall voltage with the sample rotated by $0^{\circ}$ and $180^{\circ}$ with respect to the field. The Hall coefficient is then given by $R_{H}=\left(V_{0}-V_{180}\right) t / 2 I B$, where $B$ is the magnetic field, $t$ is the sample thickness, and $I$ is the current. This method eliminates the MR of the sample, and the offset voltage from $\rho_{x x}$ due to contact misalignment. Where $R_{H}$ was measured as a function of field, this was swept to both positive and negative values and $R_{H}(B)$ determined from $V_{B}$ $-V_{-B} \cdot$

TEP measurements were made by the "toggled" heating method. ${ }^{14,15}$ Two $25-\mu \mathrm{m}$ chromel-alumel thermocouples, attached to the sample with small blobs of silver paint, measure both the thermal emf and temperature gradient, ensuring that these are measured between the same two points. The sample is first stabilized at the measurement temperature, a small thermal gradient is applied, and the resulting thermal emf measured. The thermal gradient is then reversed, allowing slowly changing thermal emf's in the cryostat wires to be

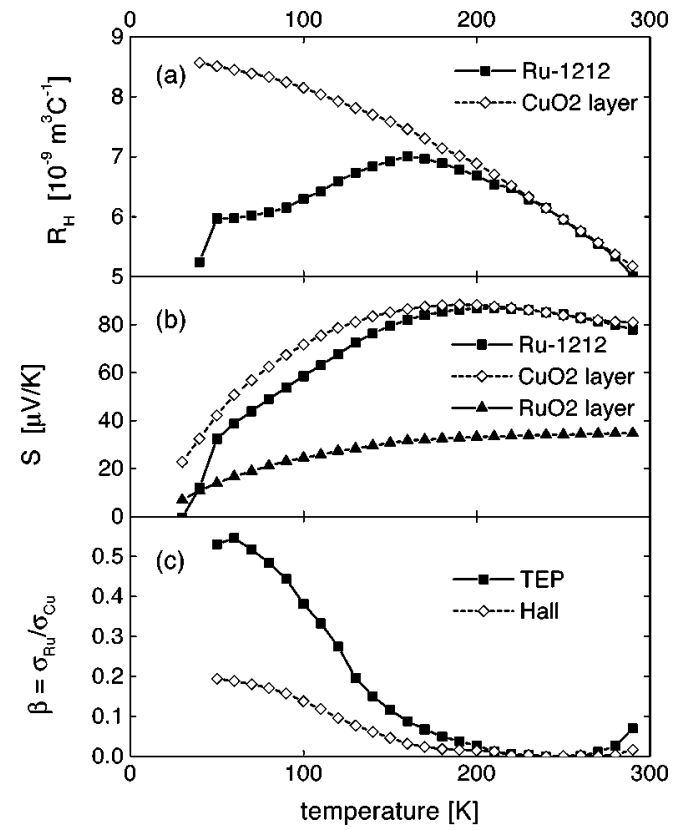

FIG. 1. (a) Hall-effect and (b) thermopower data for $\mathrm{RuSr}_{2} \mathrm{GdCu}_{2} \mathrm{O}_{8}$, together with estimated values of the $\mathrm{CuO}_{2}$ and $\mathrm{RuO}_{2}$ layer properties as described in the text. Panel (c) shows the ratio $\beta=\sigma_{\mathrm{Ru}} / \sigma_{\mathrm{Cu}}$ calculated in the two-band model, assuming $\sigma_{\mathrm{Ru}} \simeq 0$ well above $T_{\text {mag }}$.

nulled out. A "rest state" was added whereby both ends of the sample were heated at half power, providing two extra measurement points. Adding this state keeps the total power dissipation into the stage constant, avoiding fluctuation of its temperature when the heater currents are changed.

\section{RESULTS}

\section{A. Transport measurements on pure $\mathrm{RuSr}_{2} \mathrm{GdCu}_{2} \mathrm{O}_{8}$}

Hall-effect, thermopower, and resistivity data for undoped $\mathrm{RuSr}_{2} \mathrm{GdCu}_{2} \mathrm{O}_{8}$ are shown in Fig. 1. The room-temperature value of the TEP implies a hole concentration $p_{\mathrm{Cu}}$ of 0.06-0.07 holes/Cu, ${ }^{16}$ while its temperature dependence is typical of other high- $T_{c}$ materials, with the exception of the unusual linear temperature dependence below $T_{\text {mag }}$. The overall magnitude and temperature dependence of the Hall coefficient is consistent with a doping level, $p_{\mathrm{Cu}}$, of $\simeq 0.07$ holes $/ \mathrm{Cu}$, as inferred from the room-temperature TEP. $R_{H}$ displays a high- $T_{c}$-like temperature dependence well above $T_{\text {mag }}$. However, below about $170 \mathrm{~K}$ there is an anomalous downturn in $R_{H}$ which is not seen in typical high$T_{c}$ data. The so-called "anomalous" Hall effect observed in magnetic materials has been measured and discounted as the cause of this downturn. ${ }^{17}$ Alternatively, it is due to charge delocalization in the $\mathrm{RuO}_{2}$ plane occurring near the magnetic transition, or due to charge transfer into the $\mathrm{CuO}_{2}$ layers. It will be shown that a two-band model, with a localized to itinerant transition occurring at $T_{\text {mag }}$ in the $\mathrm{RuO}_{2}$ layer, can explain both these and the TEP data.

\section{The conductivity of the $\mathrm{Ru} \mathrm{O}_{2}$ layer}

The bands in this model are those formed by carriers in the $\mathrm{Cu}$ and $\mathrm{Ru}$ orbitals; the overall TEP and Hall effect are 
given by the sum of the $\mathrm{CuO}_{2}$ and $\mathrm{RuO}_{2}$ layer values, weighted by the layer conductivities as follows: ${ }^{18,19}$

$$
\begin{gathered}
R_{H}=\frac{R_{H}^{\mathrm{Ru}}\left(\sigma_{x x}^{\mathrm{Ru}}\right)^{2}+R_{H}^{\mathrm{Cu}}\left(\sigma_{x x}^{\mathrm{Cu}}\right)^{2}}{\left(\sigma_{x x}^{\mathrm{Ru}}+\sigma_{x x}^{\mathrm{Cu}}\right)^{2}}, \\
S=\frac{S^{\mathrm{Ru}} \sigma_{x x}^{\mathrm{Ru}}+S^{\mathrm{Cu}} \sigma_{x x}^{\mathrm{Cu}}}{\sigma_{x x}^{\mathrm{Ru}}+\sigma_{x x}^{\mathrm{Cu}}} .
\end{gathered}
$$

With some reasonable estimates of the $\mathrm{RuO}_{2}$ and $\mathrm{CuO}_{2}$ layer properties, it is possible to use this model and the measured room-temperature Hall effect and TEP to place a limit on the conductivity of the $\mathrm{RuO}_{2}$ layer. To do this we assume that the Hall coefficient of the $\mathrm{RuO}_{2}$ layer is approximately zero (the maximum value observed in other two-dimensional $\mathrm{Ru}$ oxides studied to date is $\left.5 \times 10^{-10} \mathrm{~m}^{3} \mathrm{C}^{-1}\right) .{ }^{20-22}$ With this assumption in Eq. (1), the conductivity of the $\mathrm{RuO}_{2}$ layer may be estimated from

$$
\begin{gathered}
\frac{\sigma_{x x}^{\mathrm{Ru}}}{\sigma_{x x}^{\mathrm{Cu}}}=\sqrt{\frac{R_{H}^{\mathrm{Cu}}}{R_{H}}}-1, \\
R_{H}^{\mathrm{Ru}} \ll R_{H}^{\mathrm{Cu}} ; \quad \sigma_{x x}^{\mathrm{Ru}}<\sigma_{x x}^{\mathrm{Cu}} .
\end{gathered}
$$

The ratio of the Hall coefficient of the $\mathrm{CuO}_{2}$ layers, $R_{H}^{\mathrm{Cu}}$, to the measured value $R_{H}$ caused by the presence of the $\mathrm{RuO}_{2}$ layer is hard to estimate due to the uncertain doping state in $\mathrm{RuSr}_{2} \mathrm{GdCu}_{2} \mathrm{O}_{8}$ and the spread of values of $R_{H}^{\mathrm{Cu}}$, for a given doping level, in the literature. ${ }^{23-25}$ Given these uncertainties, a reasonable range of values of $R_{H}^{\mathrm{Cu}} / R_{H}$ is $1-1.4$, giving $\sigma_{x x}^{\mathrm{Ru}}$ in the range $(0-0.18) \sigma_{x x}^{\mathrm{Cu}}$. The summary of $R_{H}^{\mathrm{Cu}}$ values in the review by Cooper and Loram ${ }^{26}$ would favor the low end of this range.

For this range of conductivity in the $\mathrm{RuO}_{2}$ layer, Eq. (2) predicts that the measured net TEP lies some $0-8 \mu \mathrm{V} \mathrm{K}^{-1}$ below the intrinsic $\mathrm{CuO}_{2}$ layer value, i.e., $75 \leqslant S_{290}^{\mathrm{Cu}} \leqslant 83$. It is very unlikely that $S_{290}^{\mathrm{Cu}}$ lies in the upper half of this range: a value of $S=83 \mu \mathrm{V} \mathrm{K}^{-1}$ would imply an extremely small hole concentration for which a $T_{c}$ as high as $46 \mathrm{~K}$ would be extraordinary.

Having placed a limit on the conductivity one can use a two-dimensional model to determine $k_{F} l$, the product of the Fermi wave vector with the mean free path for the $\mathrm{RuO}_{2}$ layers. This quantity gives an indication as to whether the carriers are localized or itinerant and for a cylindrical Fermi surface may be written as

$$
k_{F} l=\sigma \frac{2 \pi \hbar c}{e^{2}},
$$

where $c$ is the separation of the planes. Data in the literature for the $a b$-plane resistivity of underdoped $\mathrm{YBa}_{2} \mathrm{Cu}_{3} \mathrm{O}_{7-\delta}$ films and single crystals, with $p \simeq 0.07$, give a consistent value of $1.2 \mathrm{~m} \Omega \mathrm{cm}$ at room temperature ${ }^{24,27-29}$ giving $k_{F} l_{\mathrm{Cu}}=1.3$, near the limit of localization. In fact, in only slightly more underdoped samples one sees a semiconducting upturn at low temperatures. Given the range of ratios of $\sigma_{x x}^{\mathrm{Ru}}$ to $\sigma_{x x}^{\mathrm{Cu}}$ derived from the Hall effect, $k_{F} l_{\mathrm{Ru}}=0-0.45$ at room temperature. The TEP data suggest that the true value is at the low end of this range, indicating that the carriers in the $\mathrm{RuO}_{2}$ layers are at best very poorly metallic.

\section{Temperature dependence of $\sigma_{R u}$}

Having established that the room-temperature conductivity of the $\mathrm{RuO}_{2}$ layer is close to zero, typical $S(T)$ and $R_{H}(T)$ data for high- $T_{c}$ superconductors may be scaled so that the room-temperature values match those of $\mathrm{RuSr}_{2} \mathrm{GdCu}_{2} \mathrm{O}_{8}$. The differences below $T_{\text {mag }}$ may then be used to follow $\sigma_{\mathrm{Ru}}$ as a function of temperature.

Typical Hall-effect data for the $\mathrm{CuO}_{2}$ layer have been taken from measurements on sintered Ca-doped $\mathrm{YBa}_{2} \mathrm{Cu}_{3} \mathrm{O}_{7-\delta}$, while $R_{H}^{\mathrm{Ru}}$ will be set to zero, its value in other $\mathrm{RuO}_{2}$ layer compounds being much lower than $R_{H}^{\mathrm{Cu}} \cdot{ }^{20-22}$ Typical $S^{\mathrm{Cu}}$ data are approximated by measurements on sintered $\mathrm{YBa}_{2} \mathrm{Cu}_{3} \mathrm{O}_{7-\delta}$ with $\delta=0.53,{ }^{26}$ multiplied by 1.12 to match the high-temperature $\mathrm{RuSr}_{2} \mathrm{GdCu}_{2} \mathrm{O}_{8}$ data. Finally, $S^{\mathrm{Ru}}$ is approximated by data measured on a sintered sample of $\mathrm{SrRuO}_{3}$, which displays a magnitude and temperature dependence similar to that of $\mathrm{Sr}_{2} \mathrm{RuO}_{4} \cdot{ }^{30}$ All these data are shown in Fig. 1, together with the resulting $\beta(T)$ $=\sigma_{\mathrm{Ru}} / \sigma_{\mathrm{Cu}}$ calculated from Eqs. (1) and (2).

Given the uncertainties in the approximated $\mathrm{RuO}_{2}$ and $\mathrm{CuO}_{2}$ layer properties the two $\beta(T)$ curves calculated independently from the drops in $S(T)$ and $R_{H}(T)$ agree well qualitatively. If TEP data for a sample of $20 \%$ Sn-doped $\mathrm{RuSr}_{2} \mathrm{GdCu}_{2} \mathrm{O}_{8}$, in which we shall argue that $\sigma_{\mathrm{Ru}}$ is strongly suppressed below $T_{m a g}$, are used to approximate $S^{\mathrm{Cu}}$, the agreement is also quantitative. Because the TEP is a less sensitive function of $\beta$ than the Hall-effect, the difference between $S^{1212}$ and $S^{\mathrm{Cu}}$ is quite small compared with that between $R_{H}^{1212}$ and $R_{H}^{\mathrm{Cu}}$. Thus the value of $\beta$ calculated from the TEP data is more sensitive to inaccuracy in the assumed $S^{\mathrm{Cu}}$ data. This explains why using the (only slightly different) $20 \%$ Sn-doped data to approximate $S^{\mathrm{Cu}}(T)$ results in a better match to $\beta(T)$ calculated from the Hall-effect. Whichever data are used, the results show a rapid rise in the relative conductivity of the $\mathrm{RuO}_{2}$ layer below $150 \mathrm{~K}$, to $\sim 0.3 \sigma_{\mathrm{Cu}}$ or higher.

\section{B. Transport measurements on substituted $\mathrm{RuSr}_{2} \mathrm{GdCu}_{2} \mathrm{O}_{8}$}

\section{Sn-doped $\mathrm{RuSr}{ }_{2} \mathrm{GdCu} \mathrm{O}_{2}$}

The diamagnetic $\mathrm{Sn}^{4+}$ ion substitutes for Ru in solid solution, and is slightly larger in size than $\mathrm{Ru}^{4+15+}$. The effects of doping the Ru site are of extreme interest given the current debate regarding the spin and charge configuration of the Ru ions. ${ }^{3-33}$

We note that the Sn-doped samples studied here were from two sources prepared with slightly different annealing strategies. Comparison of their sample resistivities is therefore not necessarily meaningful, as annealing strongly affects the grain-boundary conductivity of $\mathrm{RuSr}_{2} \mathrm{GdCu}_{2} \mathrm{O}_{8} \cdot{ }^{10}$ In general, the resistivity of sintered high- $T_{c}$ materials is also affected by sample density. ${ }^{34,35}$

For the $2.5 \%$ and $7.5 \%$ samples the resistivity (Fig. 2) is metallic, and similar in magnitude to the undoped sample. 


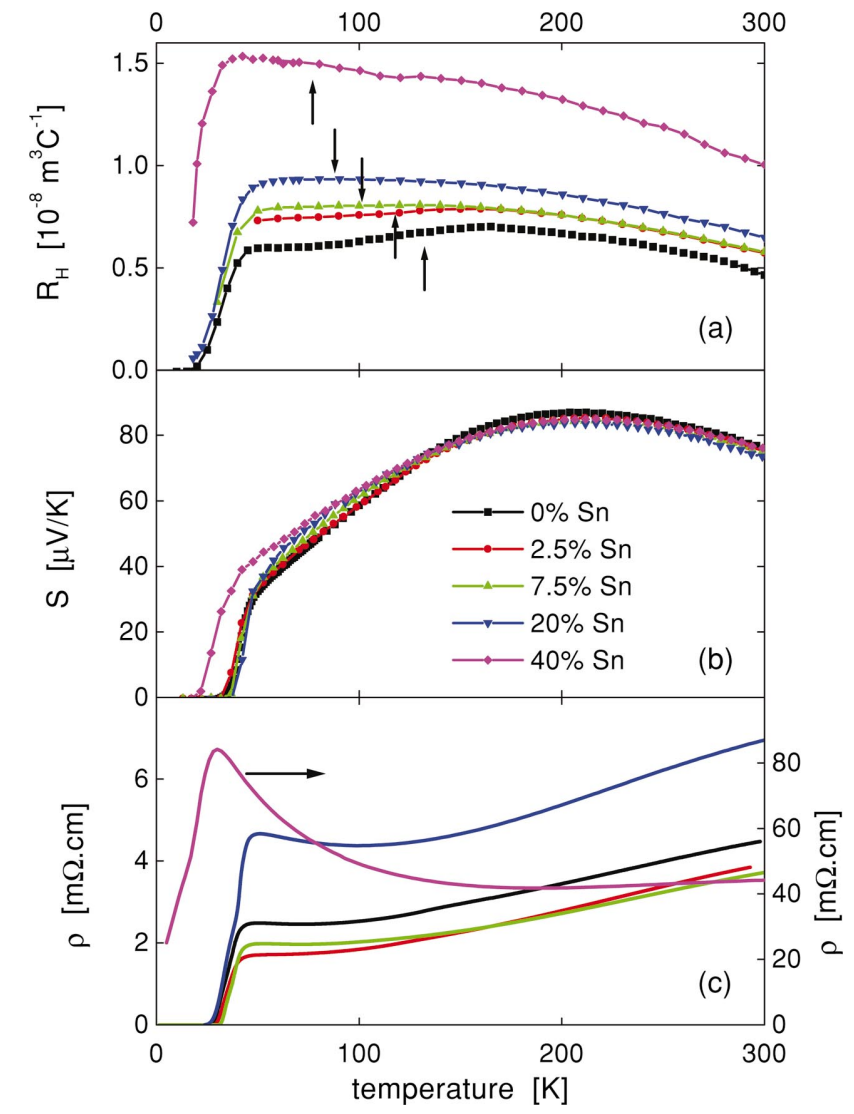

FIG. 2. (Color) (a) Hall-effect, (b) thermopower, and (c) resistivity data for Sn-doped samples of $\mathrm{RuSr}_{2} \mathrm{GdCu}_{2} \mathrm{O}_{8}$.

The 20\% sample has a higher resistivity and shows a small semiconducting upturn at low temperatures, while both the magnitude and upturn are far larger for the $40 \%$ sample. Estimating $T_{c}$ from the onset of the resistive transition reveals a gradual increase from $40.5 \mathrm{~K}$ for the $2.5 \%$ sample to $43.5 \mathrm{~K}$ for the $20 \%$ sample, while the $40 \%$ sample has a reduced $T_{c}$ of just $30 \mathrm{~K}$.

The TEP $S(T)$ and Hall effect $R_{H}(T)$ are much less affected by grain boundaries than the resistivity. In conventional high- $T_{c}$ materials they closely reflect bulk $\mathrm{CuO}_{2}$ layer properties in conventional high- $T_{c}$ materials. 35,36

The Hall-effect data show a slow and monotonic decrease in $T_{c}$ with increasing $\mathrm{Sn}$ concentration, but it should be remembered that these data were taken in a field of $10 \mathrm{~T}$ and only partly reflect the zero-field $T_{c}$. The vertical arrows in Fig. 2 (and in subsequent figures) show the location of the magnetic transition. The TEP data show that $T_{c}$ (defined by the maximum in the derivative) rises by $\sim 4 \mathrm{~K}$ in going from the $2.5 \%$ sample to the $20 \%$ sample, in good agreement with the resistivity data. The $40 \%$ sample shows a much lower transition temperature, both in $R_{H}$ and $S$. The increase in $T_{c}$ with $\mathrm{Sn}$ concentration is attributed to a transfer of holes into the $\mathrm{CuO}_{2}$ layer, ${ }^{37}$ though we observe a smaller increase than the $\sim 12 \mathrm{~K}$ reported previously. ${ }^{37,38}$ In the earlier studies $T_{c}$ was defined from the resistivity onset, and the $T_{c}$ values obtained for low doping levels were significantly lower, possibly due to granularity.
On examining the temperature and doping dependence of the normal-state properties, one immediately observes that the room-temperature TEP $S_{290}$ is little changed by the addition of Sn. This result is strange given the rise of $\sim 4 \mathrm{~K}$ in $T_{c}$ as the doping level is increased to $20 \%$. The change in the Hall effect is also counterintuitive: the $30 \%$ increase in going from 0 to $20 \%$ Sn would normally indicate a decrease in hole concentration. This apparent paradox is resolved when it is noticed that the anomalous drop in $R_{H}$ below $T_{\text {mag }}$ is diminished in the $2.5 \%$ and $7.5 \%$ samples, and is absent in the 20\% sample: as the $\mathrm{Sn}$ concentration is increased the $\mathrm{RuO}_{2}$ layer becomes well localized below $T_{m a g}$, reflecting significantly reduced conductivity at all temperatures. The changes in $R_{H}(290)$ and $S_{290}$ may then be explained quite simply: the introduction of Sn dopes a few extra holes into the $\mathrm{CuO}_{2}$ layer, increasing $p_{\mathrm{Cu}}$ and raising $T_{c}$ by $\sim 4 \mathrm{~K}$, but also drives the $\mathrm{RuO}_{2}$ layer more insulating. Thus while $R_{H}^{\mathrm{Cu}}$ probably decreases slightly, the overall Hall-effect increases as the $\mathrm{RuO}_{2}$ layer no longer provides a parallel conduction pathway. The slight increase in $p_{\mathrm{Cu}}$, which would normally decrease the measured TEP, is balanced by the decreasing $\sigma_{\mathrm{Ru}}$, which removes the reduction of the TEP by the $\mathrm{RuO}_{2}$ layer, leaving it relatively unchanged overall. Certainly, the increase in doping is far smaller than one would expect from substituting $\mathrm{Sn}^{4+}$ for $\mathrm{Ru}^{5+}$, suggesting that the mean valency of the $\mathrm{Ru}$ ion is less than $5+$. This conclusion is supported by recent $\mathrm{x}$-ray-absorption near-edge spectroscopy (XANES) measurements. ${ }^{33}$

The reduction in the room-temperature $R_{H}$ of pure $\mathrm{RuSr}_{2} \mathrm{GdCu}_{2} \mathrm{O}_{8}$, due to conductivity in the $\mathrm{RuO}_{2}$ layer, was estimated to be of the order of $30 \%$. This is entirely consistent with the rise in $R_{H}$ observed as the $\mathrm{Sn}$ concentration is increased to $20 \%$, assuming that $\sigma_{\mathrm{Ru}} \rightarrow 0$. The $40 \% \mathrm{Sn}-$ doped sample does not fit well into this picture, having a much larger $R_{H}$ at all temperatures. Given the much larger resistivity of this sample and its drastically reduced $T_{c}$, it is possible that some $\mathrm{Sn} \leftrightarrow \mathrm{Cu}$ substitution has occurred, reducing the $\mathrm{CuO}_{2}$ layer doping state, or that there are significant impurities present.

\section{2. $\mathrm{Nb}$-doped $\mathrm{RuSr}{ }_{2} \mathrm{GdCu}_{2} \mathrm{O}_{8}$}

$\mathrm{Nb}$ also substitutes for $\mathrm{Ru}$ in the $\mathrm{RuSr}_{2} \mathrm{GdCu}_{2} \mathrm{O}_{8}$ structure, but has a dramatically different effect on the transport properties. In contrast to the $\mathrm{Sn}$ ion, which has a charge of $4+, \mathrm{Nb}$ is believed to substitute in its usual $5+$ state $^{38}$ and thus for an average Ru valency of less than 5 will remove holes from the system, further underdoping it. The roomtemperature TEP bears this out, showing a large increase proportionate with $\mathrm{Nb}$ doping (see Fig. 3) and confirming that the $\mathrm{CuO}_{2}$ layer is progressively underdoped by the substitution of $\mathrm{Nb}$. This conclusion is supported by the commensurate increase in the Hall effect and the rapid reduction of $T_{c}$, which is $19 \mathrm{~K}$ for the $10 \%$ sample and below $1.5 \mathrm{~K}$ (if present at all) in the $20 \%$ sample.

The effect of $\mathrm{Nb}$ doping on the Ru layer is less clear. The Hall effect of the $10 \%$ sample displays a maximum near $T_{\text {mag }}$, suggesting increased $\mathrm{RuO}_{2}$ conductivity below this temperature, but the drop is not as clear as in the undoped 


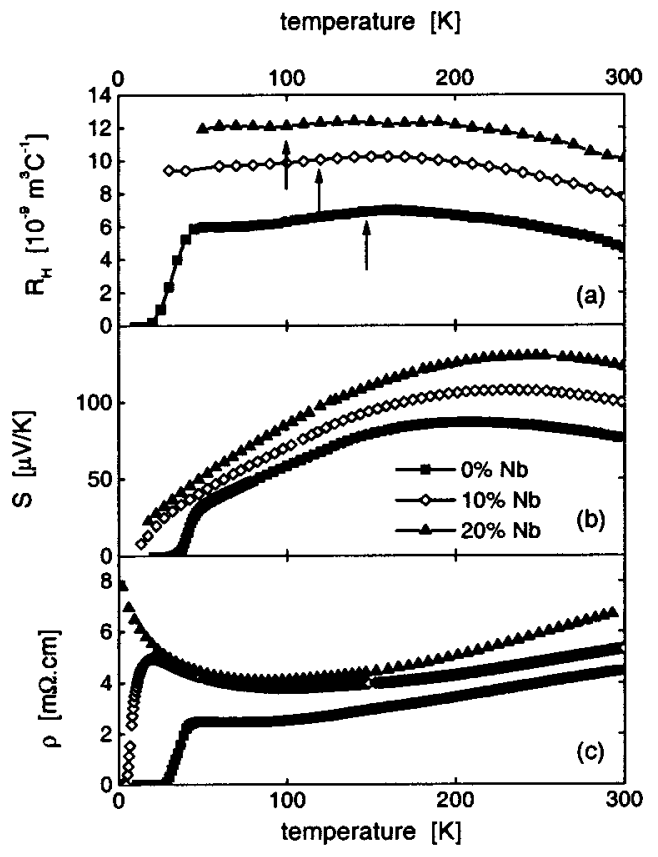

FIG. 3. (a) Hall-effect, $R_{H}(T)$, (b) thermopower, $S(T)$, and (c) resistivity, $\rho(T)$, data for $\mathrm{Nb}$-doped $\mathrm{RuSr}_{2} \mathrm{GdCu}_{2} \mathrm{O}_{8}$.

sample. For $20 \% \mathrm{Nb} \leftrightarrow \mathrm{Ru}$ substitution $R_{H}$ rises to a more or less constant value of $1.2 \times 10^{-8} \mathrm{~m}^{3} \mathrm{C}^{-1}$ below $200 \mathrm{~K}$, and there is no sign of a significant change at $T_{\text {mag }}$. The conclusion from the TEP and Hall-effect data, then, is that the transition from localized to itinerant behavior of the $\mathrm{RuO}_{2}$ layer is suppressed by the addition of $\mathrm{Nb}$, as it is by the addition of Sn.

The resistivity, on the other hand, shows surprisingly little difference between the $10 \%$ and $20 \%$ samples-in fact the residual resistivity (extrapolated from the linear hightemperature data) actually decreases. A possible scenario consistent with this result is that the $\mathrm{RuO}_{2}$ layer becomes more itinerant both above and below $T_{\text {mag }}$ as the $\mathrm{Nb}$ level is increased. However, if this were the case, the increased $\sigma_{\mathrm{Ru}}$ would be expected to suppress both $R_{H}$ and $S$ below the $\mathrm{CuO}_{2}$ plane values. In fact, for $T_{c}=19 \mathrm{~K}$ and $T_{c}^{\max }$ $\approx 100 \mathrm{~K}$, the universal relationship between $S_{290}$ and $T_{c}$ predicts $S_{290}^{\mathrm{Cu}} \sim 100 \mu \mathrm{V} \mathrm{K}^{-1}$, as observed. Thus, while the increased $S$ and $R_{H}$ and the reduced $T_{c}$ are consistent with a reduced hole concentration in the $\mathrm{CuO}_{2}$ layer and a localized $\mathrm{RuO}_{2}$ layer, the relatively good conductivity of the $20 \% \mathrm{Nb}-$ doped sample is not. One possible explanation is that the behavior of the resistivity is extrinsic to the bulk in the $20 \%$ $\mathrm{Nb}$ sample, resulting from either increased grain-boundary conductivity, or increased sample density.

\section{Ce-doped $\mathrm{RuSr}_{2} \mathrm{GdCu}_{2} \mathrm{O}_{8}$}

Unlike $\mathrm{Nb}$ and $\mathrm{Sn}$, which substitute for $\mathrm{Ru}, \mathrm{Ce}$ substitutes for $\mathrm{Gd}$ in the layer separating the two $\mathrm{CuO}_{2}$ planes, and so would be expected to affect these more than the $\mathrm{RuO}_{2}$ layers from which it is relatively remote. The $\mathrm{Ce}$ ion is expected to be in the $4+$ state in $\mathrm{RuSr}_{2} \mathrm{GdCu}_{2} \mathrm{O}_{8}$, as it is in the struc-

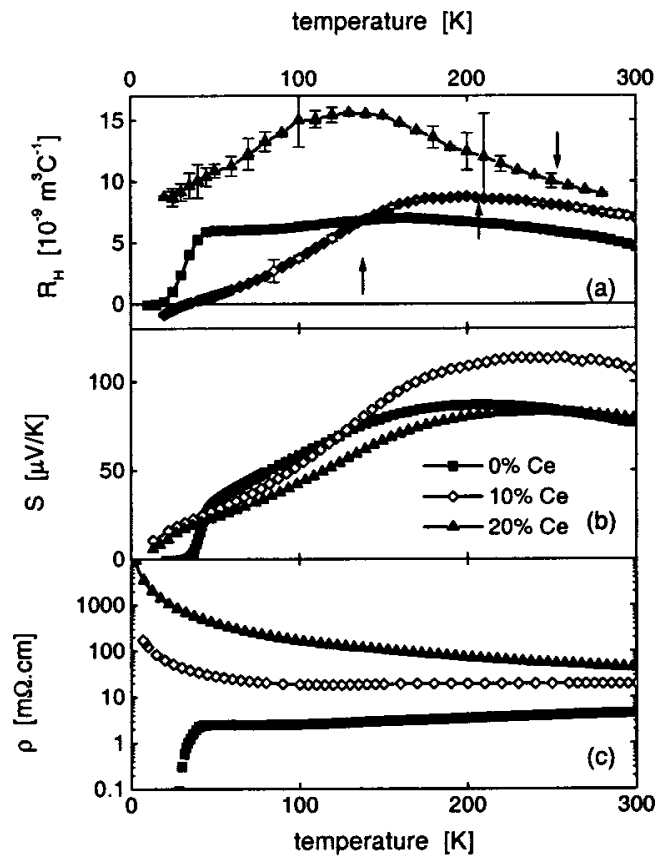

FIG. 4. (a) Hall-effect, $R_{H}(T)$, thermopower, $S(T)$, and resistivity, $\rho(T)$, data for Ce-doped $\mathrm{RuSr}_{2} \mathrm{GdCu}_{2} \mathrm{O}_{8}$. The large error bars shown on the $20 \%$ Ce Hall data points result from the exceptionally large resistivity of the sample making balancing difficult at low temperatures. The error bars shown on the $10 \% \mathrm{Ce}$ data points are more typical.

turally similar compound $\mathrm{RuSr}_{2}\left(\mathrm{Gd}_{1+x} \mathrm{Ce}_{1-x}\right) \mathrm{Cu}_{2} \mathrm{O}_{10} ;{ }^{39}$ hence its substitution for $\mathrm{Gd}^{3+}$ should further underdope the material.

Two samples (10\% and $20 \% \mathrm{Ce} \leftrightarrow \mathrm{Gd}$ ) were measured, and, of all the doped samples studied, these exhibit the most remarkable and revealing transport properties: a large drop in $R_{H}$ below $T_{\text {mag }}$ (in fact becoming negative in the $10 \%$ sample below $\sim 30 \mathrm{~K}$ ), and a large TEP at room temperature which, like the Hall-effect, drops very rapidly below $T_{\text {mag }}$. These data are shown in Fig. 4, along with the resistivities of the two samples.

We note first that, as with other electron doping substitutions ( $\mathrm{Ce}$ for $\mathrm{Gd}$, La for $\mathrm{Sr}$, and hydrogen doping), $T_{\text {mag }}$ is driven upwards. This appears to reflect an increasing $\mathrm{Ru}^{4+}$ fraction. The $10 \%$ Ce sample will be dealt with first. As with the undoped sample, the departure from cupratelike properties below $T_{\text {mag }}$ indicates a transition from localized to itinerant behavior in the $\mathrm{RuO}_{2}$ layer. In this case, however, the room-temperature TEP $S_{290}=110 \mu \mathrm{V} \mathrm{K}^{-1}$ indicates a much lower $\mathrm{CuO}_{2}$ layer carrier concentration of $p$ $\sim 0.03$ holes $/ \mathrm{Cu}$. This is consistent with the increased Hall coefficient, which is probably still depressed from the true $\mathrm{CuO}_{2}$ value by residual conductivity in the $\mathrm{RuO}_{2}$ layer, and the large resistivity with its insulating upturn at low temperature. Having concluded that $p$, and hence $\sigma_{\mathrm{Cu}}$, is much lower than in the undoped sample, the reason for the dramatic effects seen in $R_{H}$ and $S$ below $T_{\text {mag }}$ becomes clear: the ratio $\sigma_{\mathrm{Ru}} / \sigma_{\mathrm{Cu}}$ is much larger in the Ce-doped sample at low temperature, allowing the intrinsic $\mathrm{RuO}_{2}$ layer properties to dominate the behavior. 


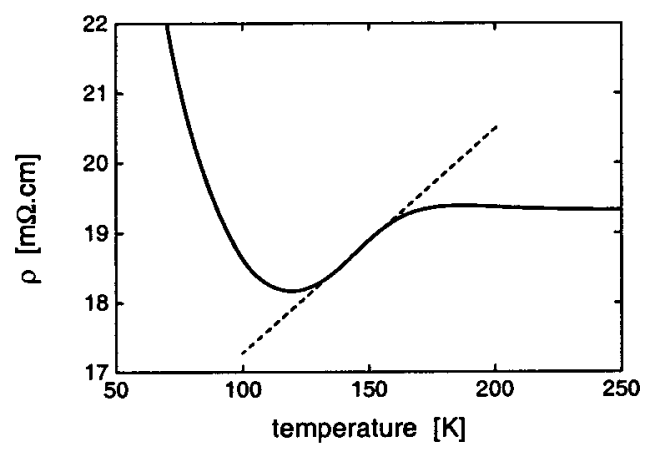

FIG. 5. Enlarged view of the resistivity of the $10 \%$ Ce-doped $\mathrm{RuSr}_{2} \mathrm{GdCu}_{2} \mathrm{O}_{8}$ sample showing $T$-linear resistivity below $T_{\text {mag }}$.

The effect of changes in $\sigma_{\mathrm{Ru}} / \sigma_{\mathrm{Cu}}$ is greater for $R_{H}$ than $S$, but for these samples the increased $\sigma_{\mathrm{Ru}}$ depresses the $S_{\mathrm{Cu}}$ contribution to the total TEP so much that $S_{\mathrm{Ru}}$ dominates below $50 \mathrm{~K}$. The basis for this assertion is the double maximum in $d S / d T$ : initially, at low temperatures, $S(T)$ follows a curve reasonably consistent with the TEP of $\mathrm{SrRuO}_{3}$. This contribution is trending towards saturation at a value of $\sim 40 \mu \mathrm{V} \mathrm{K}^{-1}$; however, above $50 \mathrm{~K}$ increasing $\sigma_{\mathrm{Cu}}$ allows $S_{\mathrm{Cu}}$ to contribute, and the overall TEP then rises more rapidly.

The same qualitative treatment may be applied successfully to the Hall-effect data, though in order to explain the negative values below $\sim 30 \mathrm{~K}$ it is necessary to assume a negative Hall coefficient for the $\mathrm{RuO}_{2}$ layer of around -1 $\times 10^{-9} \mathrm{~m}^{3} \mathrm{C}^{-1}$. Examining typical data from the $\mathrm{Sr}_{n+1} \mathrm{Ru}_{n} \mathrm{O}_{3 n+1}$ series one finds that $R_{H}$ of $\mathrm{Sr}_{3} \mathrm{Ru}_{2} \mathrm{O}_{7}$ remains positive at all temperatures, while that of $\mathrm{Sr}_{2} \mathrm{RuO}_{4}$ becomes negative below $20 \mathrm{~K}$, but reaches just -1 $\times 10^{-10} \mathrm{~m}^{3} \mathrm{C}^{-1}$ near $1 \mathrm{~K}$. However, $\mathrm{SrRuO}_{3}$, which has the most similar ferromagnetic $\mathrm{RuO}_{2}$ layer to $\mathrm{RuSr}_{2} \mathrm{GdCu}_{2} \mathrm{O}_{8}$, has a negative $R_{H}$ below $100 \mathrm{~K}$, reaching a field-dependent value of $\sim-1 \times 10^{-9} \mathrm{~m}^{3} \mathrm{C}^{-1}$ below $60 \mathrm{~K}^{20}$ Thus the value of $R_{H}$ observed in the Ce-doped sample at low temperature is the same order of magnitude as that in $\mathrm{SrRuO}_{3}$, confirming that the $\mathrm{RuO}_{2}$ layer dominates the transport properties. It is interesting to note that, though it may not be a large effect, $\mathrm{Ce}$ substitution for $\mathrm{Gd}$ should drive the mean $\mathrm{Ru}$ valence closer to $4+$, as it is in $\mathrm{SrRuO}_{3}$.

Turning now to the resistivity, one encounters a problem: if the $\mathrm{RuO}_{2}$ layer is indeed metallic below $T_{\text {mag }}$, why does the resistivity increase so dramatically as $T \rightarrow 0$ ? There are two possible answers to this question: either both the $\mathrm{RuO}_{2}$ and $\mathrm{CuO}_{2}$ layers are at least semiconducting, but such that $\sigma_{\mathrm{Ru}} / \sigma_{\mathrm{Cu}}>1$, or it may be that insulating grain boundaries cause the upturn. The second of these scenarios seems more likely. In this case the TEP and Hall effect, being much less sensitive to intergrain connectivity, are determined by a weakly metallic intrinsic $\sigma_{\mathrm{Ru}}$. Support for this conclusion is provided by close examination of the resistivity (Fig. 5) which shows an extended metallic region below $T_{m a g}$.

This type of behavior is not uncommon in $\mathrm{RuSr}_{2} \mathrm{GdCu}_{2} \mathrm{O}_{8}$-in fact extrinsic upturns in resistivity are observed in poorly annealed undoped samples. Interestingly though, transport measurements on $\mathrm{SrRuO}_{3}$ also show a minimum in resistivity below $T_{\text {Curie }}$ in samples where there is some disorder in the $\mathrm{RuO}_{2}$ layer. ${ }^{40}$ The temperature at which the minimum occurs, and the magnitude of the upturn below it both increase with $\mathrm{RuO}_{2}$ layer disorder: in good quality films the highest-temperature minimum observed is $40 \mathrm{~K}$, coincident with the maximum residual resistivity. ${ }^{40}$

The $20 \%$ Ce-doped sample deviates nontrivially from the scenario for the $10 \%$ Ce sample: $R_{H}$ is higher at room temperature, as one would expect for even greater underdoping caused by the increase in Ce content, but $S_{290}$ is actually lower than that of the $10 \%$ sample, apparently implying an increased hole concentration. In the absence of a clear resolution we prefer not to speculate on these changes which could just arise from disorder near a solubility limit.

\section{Calculation of $\beta(T)$ in Ce-doped $\mathrm{RuSr}_{2} \mathrm{GdCu}_{2} \mathrm{O}_{8}$}

The ratio $\beta(T)=\sigma_{\mathrm{Ru}}(T) / \sigma_{\mathrm{Cu}}(T)$ may be extracted from the data for the $10 \%$ Ce-doped sample using the two-band model, as described for the undoped material. As in the previous calculation, typical $S^{\mathrm{Cu}}(T)$ and $R_{H}^{\mathrm{Cu}}(T)$ data are matched to the high-temperature $\mathrm{RuSr}_{2} \mathrm{GdCu}_{2} \mathrm{O}_{8}$ data, where $\sigma_{\mathrm{Ru}}$ is assumed to be small compared with $\sigma_{\mathrm{Cu}}$ and the overall properties reflect those of the $\mathrm{CuO}_{2}$ layer most strongly. The deviation from cupratelike behavior at lower temperatures is then used to extract the ratio $\beta(T)$.

For this sample, $S^{\mathrm{Cu}}(T)$ data were taken as 1.05 times $S(T)$ measured on a sample of underdoped sintered $\mathrm{YBa}_{2} \mathrm{Cu}_{3} \mathrm{O}_{7-\delta}$, with $\delta \simeq 0.6 .{ }^{26} R_{H}^{\mathrm{Cu}}(T)$ data were taken as 1.16 times $R_{H}$ measured on a similar sample with $\delta \simeq 0.62 .{ }^{41}$ $R_{H}$ and $S$ are particularly strong functions of doping in this region of the phase diagram: the good agreement in the values of $\delta$ required for the two sets of data to match those of $\mathrm{RuSr}_{2} \mathrm{GdCu}_{2} \mathrm{O}_{8}$ suggests that the assumption of negligible $\sigma_{\mathrm{Ru}}$ at high temperature is reasonable.

The TEP of the $\mathrm{RuO}_{2}$ layer is approximated by that of sintered $\mathrm{SrRuO}_{3}$, as before. As the Hall effect becomes negative at low temperatures in Ce-doped $\mathrm{RuSr}_{2} \mathrm{GdCu}_{2} \mathrm{O}_{8}$ taking $R_{H}^{\mathrm{Ru}} \simeq 0$, as was done for the undoped material, will not work. Instead a rough approximation to data for $\mathrm{SrRuO}_{3}$ is used, $^{20}$ which shows a field-dependent value of $\sim-1$ $\times 10^{-9}$ at $20 \mathrm{~K}$.

The measured and estimated data together with the results of the calculations are shown in Fig. 6 . Above $50 \mathrm{~K}$ there is remarkable agreement between $\beta(T)$ calculated from the TEP data $\left(\beta_{T E P}\right)$ and that calculated independently from the Hall-effect data $\left(\beta_{\text {Hall }}\right)$, lending confidence both to the model and to the estimated $R_{H}(T)$ and $S(T)$ data for the $\mathrm{RuO}_{2}$ and $\mathrm{CuO}_{2}$ layers. Below $50 \mathrm{~K}$ the agreement is not so good: $\beta_{\text {Hall }}$ carries on increasing, a direct result of $R_{H}^{1212}$ becoming very close to the estimated $R_{H}^{\mathrm{Ru}}$ at low temperatures. $S^{1212}$ does not approach the estimated $S^{\mathrm{Ru}}$ as closely, and hence $\beta_{T E P}$ does not continue to increase. Emerging clearly from these data is a large increase in $\beta$ below $T_{\text {mag }}$. At $50 \mathrm{~K} \sigma_{\mathrm{Ru}} / \sigma_{\mathrm{Cu}} \sim 1.9$, whereas for the undoped material the increase in $\sigma_{\mathrm{Ru}} / \sigma_{\mathrm{Cu}}$ is just 0.3 . The properties of the $\mathrm{RuO}_{2}$ layer dominate the overall transport properties of $\mathrm{RuSr}_{2} \mathrm{GdCu}_{2} \mathrm{O}_{8}$ below $\sim 90 \mathrm{~K}$ in this $10 \%$ Ce-doped sample. 


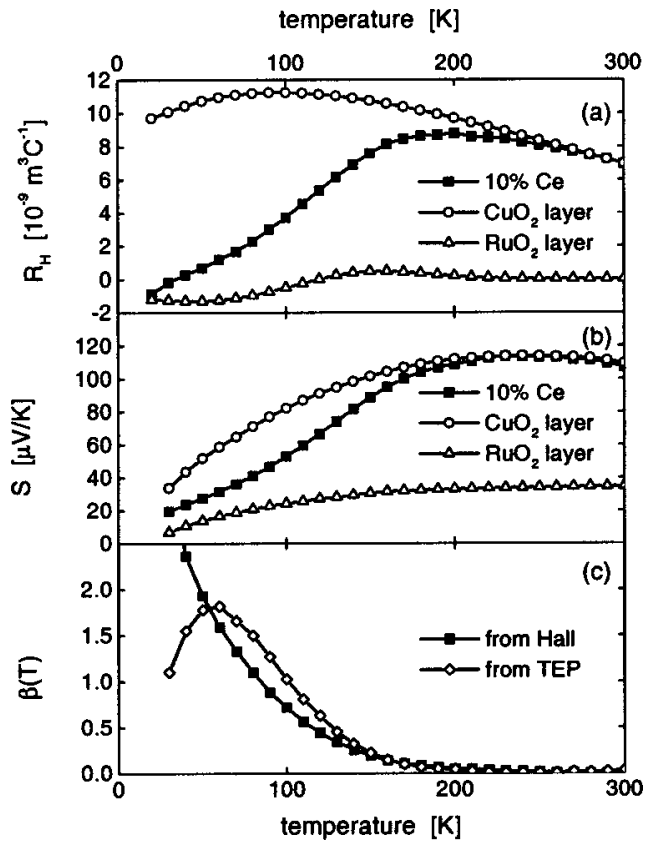

FIG. 6. (a) Hall-effect and (b) thermopower data for $\mathrm{RuSr}_{2} \mathrm{Gd}_{0.9} \mathrm{Ce}_{0.1} \mathrm{Cu}_{2} \mathrm{O}_{8}$, together with the estimated $\mathrm{RuO}_{2}$ and $\mathrm{CuO}_{2}$ layer values, as described in the text. Panel (c) shows the ratio $\beta=\sigma_{\mathrm{Ru}} / \sigma_{\mathrm{Cu}}$ calculated in the two-band model from thermopower and, independently, from the Hall effect.

\section{Other doped samples}

The remainder of the doped samples studied contained Y, Dy, and $\mathrm{Eu}$ on the Gd site, plus a 5\% Li-doped sample, in which $\mathrm{Cu}$ is substituted. The transport data for all these samples are shown in Fig. 7.

The Hall effect shows the "usual" anomalous downturn below $T_{\text {mag }}$ in all these samples. The magnitude of the downturn, due to the transition to a more itinerant $\mathrm{Ru}$ layer, is approximately constant, leading to the conclusion that doping the $\mathrm{Cu}$ and $\mathrm{Gd}$ sites does not greatly affect the localization of carriers in the $\mathrm{RuO}_{2}$ layer.

Substituting a small amount of $\mathrm{Li}^{+}$for $\mathrm{Cu}^{2+}$ causes virtually no change in the TEP, but depresses $T_{c}$ by $\approx 20 \mathrm{~K}$. The Hall effect of this sample is slightly larger than that of the undoped sample, possibly due to some cross substitution of $\mathrm{Li}$ with $\mathrm{Ru}$, depressing $\sigma_{\mathrm{Ru}}$, or a slight decrease in the $\mathrm{CuO}_{2}$ layer carrier concentration. These results are consistent with $\mathrm{Li}^{+}$acting as a pair breaker in the $\mathrm{CuO}_{2}$ layer, but with little other effect on transport properties. The rate of suppression of $T_{c}$ with $\mathrm{Li}$ substitution in $\mathrm{RuSr}_{2} \mathrm{GdCu}_{2} \mathrm{O}_{8}, \sim 4 \mathrm{~K} / \%$, is about one quarter of that observed in underdoped $\mathrm{YBa}_{2} \mathrm{Cu}_{3} \mathrm{O}_{7-\delta}(\delta=0.4)$ when either $\mathrm{Li}$ or $\mathrm{Zn}$ is substituted for $\mathrm{Cu}^{42}$ However the concentration of $\mathrm{Li}$ in the $\mathrm{RuSr}_{2} \mathrm{GdCu}_{2} \mathrm{O}_{8}$ sample studied is nominal, and the difference in the rate of suppression may simply reflect loss of $\mathrm{Li}$ by vaporization during the long synthesis and anneal.

The isovalent substitution of $\mathrm{Y}$ or Dy for $\mathrm{Gd}$ actually causes a slight decrease in the TEP of $\mathrm{RuSr}_{2} \mathrm{GdCu}_{2} \mathrm{O}_{8}$, these being the only substitutions studied to do so. The implied increase in the doping level of the $\mathrm{CuO}_{2}$ layers, presumed to arise from an ion-size effect, is confirmed by the increased

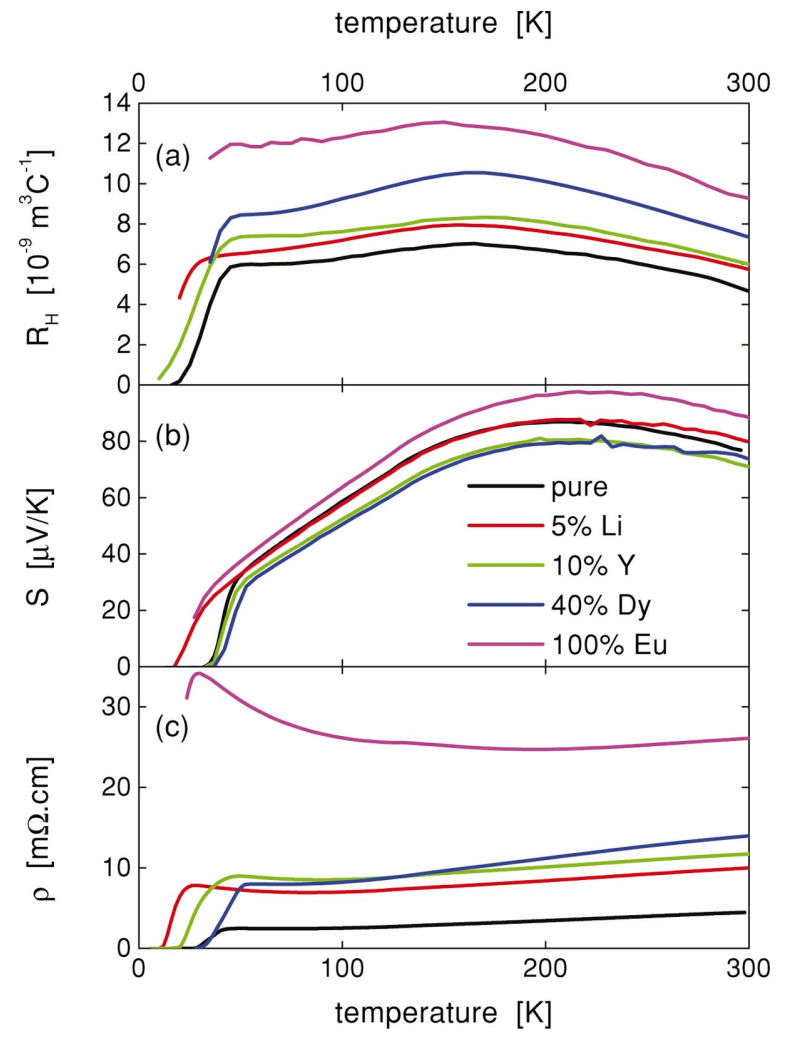

FIG. 7. (Color) (a) Hall-effect, $R_{H}(T)$, (b) thermopower, $S(T)$, and (c) resistivity, $\rho(T)$, data for $\mathrm{RuSr}_{2} \mathrm{GdCu}_{2} \mathrm{O}_{8}$ with $10 \% \mathrm{Y}, 40 \%$ Dy, and $100 \%$ Eu substituted for $\mathrm{Gd}$, or $5 \% \mathrm{Li}$ substituted for $\mathrm{Cu}$.

$T_{c}$ in these samples- $\approx 2 \mathrm{~K}(10 \% \mathrm{Y})$ and $6 \mathrm{~K}(40 \% \mathrm{Dy})$ higher than in the undoped sample as seen by both resistivity and TEP measurements. However, the magnitude of the Halleffect is larger for these samples than for the undoped ones. Having argued that the $\mathrm{CuO}_{2}$ layer is less underdoped in these two samples, this effect may only arise from a decrease in the conductivity of the $\mathrm{RuO}_{2}$ layer, partially removing the "shorting" of the $\mathrm{CuO}_{2}$ layer Hall-effect.

Full substitution of Eu for Gd causes an increase in $S_{290}$ to $90 \mu \mathrm{V} \mathrm{K}^{-1}, T_{c}$ as measured by the TEP or resistivity drops significantly, and $R_{H}$ is greatly increased. All these results suggest a drop in the $\mathrm{CuO}_{2}$ layer hole concentration, again consistent with the above-noted ion-size effect, perhaps coupled with a decrease in $\sigma_{\mathrm{Ru}}$. Consistent with this interpretation, the resistivity of this sample is much less metallic than that of the others (which are all metallic, with magnitudes two to three times the well-annealed undoped sample).

\section{DISCUSSION}

The Hall-effect and TEP data described in this paper provide strong evidence for a transition from very poorly metallic to more itinerant behavior in the $\mathrm{RuO}_{2}$ layer below $T_{\text {mag }}$. Results from substituted $\mathrm{RuSr}_{2} \mathrm{GdCu}_{2} \mathrm{O}_{8}$ samples confirm this picture. The universal relationship between $S_{290}$ and $p_{\mathrm{Cu}}$ appears to hold in $\mathrm{RuSr}_{2} \mathrm{GdCu}_{2} \mathrm{O}_{8}$ as a result of the low $\sigma_{\mathrm{Ru}}$ at room temperature, though below $T_{\text {mag }}$ both $S$ and $R_{H}$ are reduced by the shorting of the $S_{\mathrm{Cu}}$ by $\mathrm{RuO}_{2}$ layer. 
The two-band model proposed is successful in explaining most of the existing data qualitatively: the anomalies, which lie mainly in resistivity data, are most likely due to grainboundary and density effects. The quantitative agreement is also reasonably good. The results support a picture in which the $\mathrm{RuO}_{2}$ layer in the pure compound is localized above $T_{\text {mag }}$, with $\sigma_{\mathrm{Ru}} \sim 0.1 \sigma_{\mathrm{Cu}}$, but becomes more conducting below $T_{\text {mag }}$, mirroring the behavior of other ruthenates.

The transition from localized to itinerant $\mathrm{RuO}_{2}$ layer behavior at $T_{m a g}$ in the undoped compound may be modified by substituting $\mathrm{Ru}$ with $\mathrm{Sn}$ or $\mathrm{Nb}$. Sn increases the doping level of the $\mathrm{CuO}_{2}$ layers, raising $T_{c}$ and suppressing $T_{\text {mag }}$, and simultaneously drives the $\mathrm{RuO}_{2}$ layer more insulating. $\mathrm{Nb}$ underdopes the $\mathrm{CuO}_{2}$ layers, lowering $T_{c}$, and also appears to drive the $\mathrm{RuO}_{2}$ layer insulating, though the $20 \%$ sample does not show the expected semiconducting resistivity. These results imply an initial $\mathrm{Ru}$ valence lying between $4+$ and $5+$, in agreement with XANES data which may be modeled as an admixture of $40 \% \mathrm{Ru}^{4+}$ and $60 \% \mathrm{Ru}^{5+} .33$

As might be expected, doping of the $\mathrm{Cu}$ site has little effect on $T_{\text {mag }}$ or the transport properties of the $\mathrm{RuO}_{2}$ layer. $\mathrm{Li}^{+}$acts as a pair-breaking impurity in the $\mathrm{CuO}_{2}$ layer and causes a depression of $T_{c}$ in line with its behavior in other cuprates. Isovalent doping of the $\mathrm{Gd}$ site with other lanthanide elements changes the $\mathrm{CuO}_{2}$ layer doping level, with a remarkably strong variation in $T_{c}$. This appears to be a ion-size doping effect. Altervalent substitution of $\mathrm{Ce}$ for $\mathrm{Gd}$ rapidly reduces the doping level of the $\mathrm{CuO}_{2}$ layers and drives the material nonsuperconducting. In all but the $\mathrm{Ce}$ doped samples, the conductivity of the $\mathrm{RuO}_{2}$ layer only ever reaches a modest fraction of that of the $\mathrm{CuO}_{2}$ layer. In the $10 \%$ Ce-doped sample the more heavily underdoped $\mathrm{CuO}_{2}$ layer has an insulating upturn at low temperature, while the $\mathrm{RuO}_{2}$ layer remains more metallic, and so the ratio of their conductivities reaches at least 1.9.

\section{CONCLUSIONS}

To a first approximation the electronic properties of the $\mathrm{CuO}_{2}$ layer in $\mathrm{RuSr}_{2} \mathrm{GdCu}_{2} \mathrm{O}_{8}$ are the same as those of similar $\mathrm{CuO}_{2}$ layers in other high- $T_{c}$ cuprate superconductors in all respects. This conclusion is supported by the resistivity,
TEP, and Hall-effect data presented here, and by results on the specific-heat jump at $T_{c}$. On a more detailed level, magnetotransport measurements reveal an interaction between the carriers in these layers and the magnetization of the $\mathrm{RuO}_{2}$ layer. This interaction, with an energy which would seem to be of the same order as the SC energy gap ${ }^{10}$ is not sufficient to destroy superconductivity.

The electronic properties of the $\mathrm{RuO}_{2}$ layer appear to bear a remarkable similarity to those observed in the ruthenate $\mathrm{SrRuO}_{3}$. At room temperature the conductivity of the layer is perhaps $10 \%$ of that of the $\mathrm{CuO}_{2}$ layer, with $k_{F} l_{\mathrm{Ru}} \approx 0.2$, indicating very badly metallic or localized behavior. Below $T_{\text {mag }}$ the conductivity of the layer rises significantly - by at least $0.3 \sigma_{\mathrm{Cu}}$. This increase raises the weighting of the $\mathrm{RuO}_{2}$ layer properties relative to those of the $\mathrm{CuO}_{2}$ layer in the admixture that determines the overall transport properties of $\mathrm{RuSr}_{2} \mathrm{GdCu}_{2} \mathrm{O}_{8}$. As the Hall-effect and TEP of the $\mathrm{RuO}_{2}$ layer are both considerably smaller than those in the $\mathrm{CuO}_{2}$ layer the result is a drop in both $R_{H}$ and $S$ below $T_{\text {mag }}$. In pure $\mathrm{RuSr}_{2} \mathrm{GdCu}_{2} \mathrm{O}_{8}$, and also in most of the substituted variants studied, $\sigma_{\mathrm{Ru}}$ remains lower than $\sigma_{\mathrm{Cu}}$ over the whole temperature range. For the Ce-doped samples studied, however, the $\mathrm{CuO}_{2}$ layer becomes insulating at low temperatures, allowing the poorly metallic $\mathrm{RuO}_{2}$ layer to dominate the conductivity, and its intrinsic transport properties to show strongly in the overall $R_{H}$ and $S$ of the material.

The two-band model of parallel conduction in the $\mathrm{RuO}_{2}$ and $\mathrm{CuO}_{2}$ layers has been very successful in modeling the transport properties observed in all the $\mathrm{RuSr}_{2} \mathrm{GdCu}_{2} \mathrm{O}_{8}$ samples studied, and it has been possible to describe well the effects of doping the different atomic sites. The inferred mixed valency of $\mathrm{Ru}$, together with the onset of itinerancy at the magnetic transition, suggests a possible role of a doubleexchange mechanism in the magnetic interactions but also raises the possibility of charge ordering in these compounds at appropriate doping levels.

\section{ACKNOWLEDGMENTS}

This work was supported by the U.K. Engineering and Physical Sciences Research Council and the New Zealand Marsden Fund for research (JLT) and travel funds (CB).
${ }^{1}$ L. Bauernfeind, W. Widder, and H.F. Braun, J. Low Temp. Phys. 105, 1605 (1996).

${ }^{2}$ J.L. Tallon, C. Bernhard, and J.W. Loram, J. Low Temp. Phys. 117, 823 (1999).

${ }^{3}$ C. Bernhard, J.L. Tallon, Ch. Niedermayer, Th. Blasius, A. Golnik, E. Bruecher, R.K. Kremer, D.R. Noakes, C.E. Stronach, and E.J. Ansaldo, Phys. Rev. B 59, 14099 (1999).

${ }^{4}$ A. Fainstein, E. Winkler, and A. Butera, Phys. Rev. B 60, R12 597 (1999).

${ }^{5}$ C. Bernhard, J.L. Tallon, E. Bruecher, and R.K. Kremer, Phys. Rev. B 61, 14960 (2000).

${ }^{6}$ J.E. McCrone, J.R. Cooper, J.W. Loram, and J.L. Tallon (unpublished).
${ }^{7}$ O. Chmaissem, J.D. Jorgensen, H. Shaked, P. Dollar, and J.L. Tallon, Phys. Rev. B 61, 6401 (2000).

${ }^{8}$ J. Lynn, B. Keimer, C. Ulrich, C. Bernhard, and J.L. Tallon, Phys. Rev. B 61, R14 964 (2000).

${ }^{9}$ H. Takigawa, J. Akimitsu, H. Kawano-Furukawa, and $\mathrm{H}$. Yoshizawa, J. Phys. Soc. Jpn. 70, 333 (2001).

${ }^{10}$ J.E. McCrone, J.R. Cooper, and J.L. Tallon, J. Low Temp. Phys. 117, 1199 (1999).

${ }^{11}$ M. Pozek, A. Dulcic, D. Paar, A. Hamzic, M. Basletic, E. Tafra, G.V.M. Williams, and S. Kramer, Phys. Rev. B 65, 174514 (2002).

${ }^{12}$ Y. Tokunaga, H. Kotegawa, K. Ishida, Y. Kitaoka, H. Takagiwa, and J. Akimitsu, Phys. Rev. Lett. 86, 5767 (2001). 
${ }^{13}$ J. Tallon, C. Bernhard, M.E. Bowden, P.W. Gilberd, T. Stoto, and D.J. Pringle, IEEE Trans. Appl. Supercond. 9, 1696 (1999).

${ }^{14}$ J.R. Cooper, C. Rizzuto, and G. Olcese, J. Phys. (Paris) 32, 1136 (1971).

${ }^{15}$ R. Resel, E. Gratz, A.T. Burkov, T. Nakama, M. Higa, and K. Yagasaki, Rev. Sci. Instrum. 67, 1970 (1996).

${ }^{16}$ S.D. Obertelli, J.R. Cooper, and J.L. Tallon, Phys. Rev. B 46, 14928 (1992).

${ }^{17}$ J.E. McCrone, Ph.D. thesis, University of Cambridge, 2001.

${ }^{18}$ C.M. Hurd, The Hall Effect in Metals and Alloys (Plenum, New York, 1972).

${ }^{19}$ R.D. Barnard, Thermoelectricity in Metals and Alloys (Taylor and Francis, London, 1972).

${ }^{20}$ H.C. Yang, S.H. Liu, L.M. Wang, and H.E. Horng, J. Appl. Phys. 85, 5792 (1999).

${ }^{21}$ A.P. Mackenzie, N.E. Hussey, A.J. Diver, S.R. Julian, Y. Maeno, S. Nishizaki, and T. Fujita, Phys. Rev. B 54, 7425 (1996).

${ }^{22}$ R.S. Perry, L.M. Galvin, A.P. Mackenzie, D.M. Forsythe, S.R. Julian, S.I. Ikeda, and Y. Maeno, Physica B 284, 1469 (2000).

${ }^{23}$ B. Wuyts, V.V. Moshchalkov, and Y. Bruynseraede, Phys. Rev. B 53, 9418 (1996).

${ }^{24}$ A. Carrington, D.J.C. Walker, A.P. Mackenzie, and J.R. Cooper, Phys. Rev. B 48, 13051 (1993).

${ }^{25}$ I.R. Fisher, P.S.I.P.N. de Silva, J.W. Loram, J.L. Tallon, A. Carrington, and J.R. Cooper, Physica C 235-240, 1497 (1994).

${ }^{26}$ J.R. Cooper and J.W. Loram, J. Phys. I 6, 2237 (1996).

${ }^{27}$ Y. Sun, G. Strasser, E. Gornik, and X.Z. Wang, Physica C 223, 14 (1994).
${ }^{28}$ R. Jin and H.R. Ott, Phys. Rev. B 57, 13872 (1998).

${ }^{29}$ A. Carrington, A.P. Mackenzie, C.T. Lin, and J.R. Cooper, Phys. Rev. Lett. 69, 2855 (1992).

${ }^{30}$ H. Yoshino, K. Murata, N. Shirakawa, Y. Nishihara, Y. Maeno, and T. Fujita, J. Phys. Soc. Jpn. 65, 1548 (1996).

${ }^{31}$ G.V.M. Williams and S. Kramer, Phys. Rev. B 62, 4132 (2000).

${ }^{32}$ A. Butera, A. Fainstein, E. Winkler, and J.L. Tallon, Phys. Rev. B 63, 054442 (2001).

${ }^{33}$ R.S. Liu, L.-Y. Jang, H.-H. Hung, and J.L. Tallon, Phys. Rev. B 63, 212507 (2001).

${ }^{34}$ J.R. Cooper, S.D. Obertelli, P.A. Freeman, D.N. Zheng, J.W. Loram, and W.Y. Liang, Supercond. Sci. Technol. 4, S277 (1991).

${ }^{35}$ I.R. Fisher and J.R. Cooper, Physica C 272, 125 (1996).

${ }^{36}$ A. Carrington and J.R. Cooper, Physica C 219, 119 (1994).

${ }^{37}$ A.C. Maclaughlin and J.P. Attfield, Phys. Rev. B 60, 14605 (1999).

${ }^{38}$ A.C. Maclaughlin, V. Janowitz, J.A. McAllister, and J.P. Attfield, Chem. Commun. (Cambridge) 14, 1331 (2000).

${ }^{39}$ I. Felner, U. Asaf, C. Godart, and E. Alleno, Physica B 261, 703 (1999).

${ }^{40}$ L. Klein, J.S. Dodge, C.H. Ahn, G.J. Snyder, T.H. Geballe, M.R. Beasley, and A. Kapitulnik, Phys. Rev. Lett. 77, 2774 (1996).

${ }^{41}$ A. Carrington, Ph.D. thesis, University of Cambridge, 1993.

${ }^{42}$ J. Bobroff, W.A. MacFarlane, H. Alloul, P. Mendels, N. Blanchard, G. Collin, and J.-F. Marucco, Phys. Rev. Lett. 83, 4381 (1999). 